\title{
PHOTOMETRIC BEHAVIOUR OF FIELD RR LYRAE STARS*
}

\author{
(Abstract) \\ K. STEPIEN \\ Warsaw University Observatory, Poland
}

Three colour observations of the rising branches of 25 field RR Lyrae stars are presented. This photometry supplemented with the observations by Sturch (1966) permitted the finding of mean colour indices of a number of single-period variables. Observations of additional single-period variables with well determined light curves were included in the discussion. The unreddened colour indices were found for stronglined and weak-lined variables and then the differential blanketing effect between the two groups was removed. The results indicate that the width of the instability strip for field RR Lyrae stars is similar to that for cluster variables. Absolute magnitudes and masses of both groups were found. They are: $M_{v} \approx 0.8$ and $1.3, M \approx 0.52$ $M_{\odot}$ and $0.47 M_{\odot}$ for weak-lined and strong-lined stars respectively. A difference in mass of $0.05 M_{\odot}$ was found from a difference in transition colour index.

All of the stars from the sample discussed which are cooler than $T_{e}=6600 \mathrm{~K}$ exhibit bumps on the rising branches of their $V$ curves. $U-B$ excess is associated with the bump. It occurs $0.02 P$ earlier than the bump - at the phase of maximum compression and at the phase of the mean $V$ magnitude. The bolometric corrections were found to be very small for RR Lyrae variables.

\section{Reference}

Sturch, C.: 1966, Astrophys. J. 143, 774.

* This paper appeared in full in Acta Astronoinica 22, 175, 1972. 